\title{
MODELO MATEMÁTICO PARA O PROCESSO DE DESFOSFORAÇÃO DE FERRO-GUSA LÍQUIDO
}

Marcus Novaes Motta ${ }^{1,2}$ Luiz Fernando Andrade de Castro ${ }^{3}$

\section{Resumo}

Considerando as exigências cada vez mais rigorosas do mercado consumidor de aço, principalmente com os teores residuais de elementos indesejáveis, onde os processos de tratamento do aço são mais limitados, foi o que motivou o desenvolvimento de um modelo matemático para previsão do teor de fósforo final no tratamento em uma estação de desfosforação de ferro-gusa em panela. Assim, para o desenvolvimento do presente trabalho, foi utilizado o conceito de partição de fósforo entre o metal e a escória e o modelo foi desenvolvido através de dados industriais da Siderúrgica Alterosa com a utilização de um agente desfosforante produzido na Tecnosulfur Sistema de Tratamento de Metais Líquidos S/A. O modelo de previsão de fósforo desenvolvido mostrou ser capaz de prever com eficiência o teor de fósforo no ferro-gusa utilizando as variáveis de entrada: teor de Silício (Si) inicial do ferro-gusa, temperatura inicial do ferro-gusa e o consumo específico ( $\mathrm{kg} / \mathrm{t}$ _ferro-gusa) do agente desfosforante utilizado durante o processo de desfosforação.

Palavras-chave: Desfosforação de ferro-gusa; Partição de fósforo; Regressão linear múltipla.

\section{MATHEMATICAL MODEL FOR DEPHOSPHORIZATION PROCESS OF LIQUID PIG IRON}

\begin{abstract}
The increasingly stringent requirements of the steel consumer market, especially with the residual contents of undesirable elements, where steel treatment processes are limited, were the reason for the development of a mathematical model to predict the final phosphorus content on the pig iron pre-treatment station. Thus, for the development of the present work, the concept of partition of phosphorus between the metal and the slag was used and the model was developed through industrial data of Siderúrgica Alterosa with the use of a dephosphorizing agent produced in Tecnosulfur Sistema de Tratamento de Metais Líquidos S/A. The developed phosphorus prediction model showed to be able to efficiently predict the phosphorus content in pig iron using the input variables: Initial silicon content ( $\mathrm{Si}$ ) of the pig iron, Initial temperature of the pig iron and the specific consumption ( $\mathrm{kg} / \mathrm{ton} \_$pig iron) of the dephosphorizing agent used during the dephosphoration process.
\end{abstract}

Keywords: Dephosphorization of pig iron; Phosphorus partition; Multiple linear regression.

\section{INTRODUÇÃO}

No atual estágio de desenvolvimento da sociedade, é impossível imaginar o mundo sem o uso do aço. A produção de aço é um forte indicador do estágio de desenvolvimento econômico de um país. Seu consumo cresce proporcionalmente à construção de edifícios, execução de obras públicas, instalação de meios de comunicação e produção de equipamentos. Esses materiais já se tornaram corriqueiros no cotidiano, mas fabricá-los exige técnica que deve ser renovada de forma cíclica, por isso o investimento constante das siderúrgicas em pesquisa.

O início e o processo de aperfeiçoamento do uso do ferro representaram grandes desafios e conquistas para a humanidade [I].

A presença do fósforo na composição do aço é considerada, na maioria das vezes, como impureza, já que em alguns casos especiais ele é adicionado em forma de elemento

'Programa de Pós-graduação em Metalurgia, Universidade Federal de Minas Gerais - UFMG, Sete Lagoas, MG, Brasil.

${ }^{2}$ Tecnosulfur S/A, Sete Lagoas, MG, Brasil. E-mail: marcus.novaes@tecnosulfur.com.br

${ }^{3}$ Departamento de Engenharia Metalúrgica, Universidade Federal de Minas Gerais - UFMG, Belo Horizonte, MG, Brasil.

2176-1523 (C) 2017 Associação Brasileira de Metalurgia, Materiais e Mineração. Publicado pela ABM. Este é um artigo de acesso aberto distribuído sob os termos da licença Creative Commons CC BY-NC-ND (Attribution-NonCommercial-NoDerivs) - https:// creativecommons.org/licenses/by-nc-nd/4.0\%. 
de liga. O fósforo tem efeito negativo nas propriedades de tenacidade e particularmente na fragilização por têmpera. Além disso, quanto mais elevado o teor de fósforo maior a segregação central, levando a uma estrutura extremamente frágil no centro da placa ou lingote [2].

A Figura I mostra um balanço de massa típico de fósforo e enxofre em um alto-forno. Onde cerca de $70 \%$ de fósforo é originário a partir de minério de ferro (que inclui o sínter e as pelotas) e cerca de $20 \%$ deste origina a partir de coque e carvão [3].

Visando o atendimento das crescentes demandas do mercado consumidor de aços de baixos teores de Fósforo, já que a qualidade das matérias-primas para a fabricação do ferro-gusa está diminuindo, a siderurgia tem otimizado seus processos de fabricação de aço objetivando o atendimento da qualidade exigida no produto final atingindo a produtividade necessária do equipamento e custo competitivo. Diante disso, o pré-tratamento do ferro-gusa torna-se importante ferramenta para o setor siderúrgico no cenário atual.

O desenvolvimento do modelo de previsão de fósforo para o tratamento de desfosforação em panela foi fundamentado na termodinâmica com as equações definidas através dos dados experimentais coletados em uma planta industrial e utilizado a equação de partição de fósforo [4]:

$$
2 \underline{P}+5 \mathrm{FeO}_{(l)}+4 \mathrm{CaO} \mathrm{O}_{(s)}=\mathrm{Ca}_{4} P_{2} \mathrm{O}_{9(s)}+5 \mathrm{Fe}_{(l)}
$$

Sendo que _ representa o estado padrão correspondente ao elemento dissolvido de $1 \%$ em peso no ferro.

Considerando a simplificação hipotética da Equação I, obtem-se a Equação 2:

$$
\underline{P}+\frac{5}{2} \mathrm{FeO}_{(l)}+2 \mathrm{CaO}_{(s)}=\mathrm{Ca}_{2} \mathrm{PO}_{4,5(s)}+\frac{5}{2} \mathrm{Fe}_{(l)}
$$

A variação de energia livre é descrita pela seguinte equação 3:

$$
\Delta G=\Delta G^{0}+R T \ln Q
$$

onde:

$R=8,31(\mathrm{~J} / \mathrm{mol} . \mathrm{K})$

$T=$ temperatura $(K)$

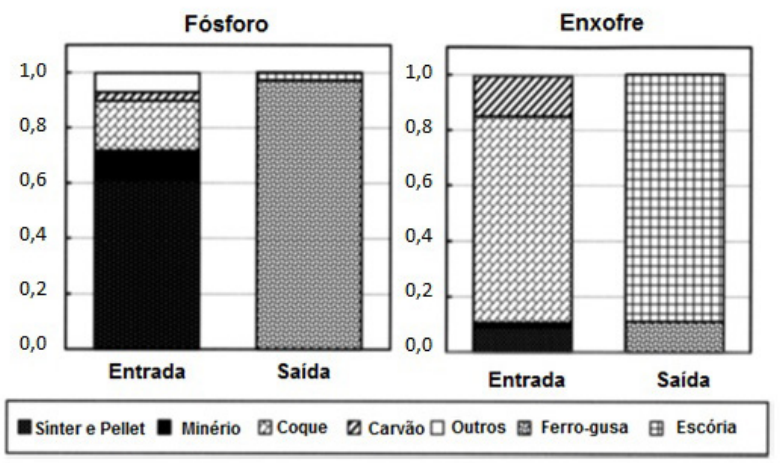

Figura I. Balanço de massa típico de fósforo e enxofre em um alto-forno. Adaptado [5].
No equilíbrio, $\Delta G=0$. Tem-se a Equação 4:

$$
\Delta G^{0}=-R T \ln K_{e q}
$$

Sendo Keq representado pela Equação 5:

$$
K_{e q}=\frac{\left(a_{\mathrm{Ca}_{2} \mathrm{PO}_{4,5}}\right)\left[a_{\mathrm{Fe}}\right]^{5 / 2}}{\left[h_{\mathrm{P}}\right]\left(a_{\mathrm{FeO}}\right)^{5 / 2}\left(a_{\mathrm{CaO}}\right)^{2}}
$$

Substituindo a Equação 5 na Equação 4, obtém-se:

$$
-\frac{\Delta G^{0}}{R T}=\ln \left[\frac{\left(a_{\mathrm{Ca}_{2} \mathrm{PO}_{4,5}}\right)\left[a_{\mathrm{Fe}}\right]^{5 / 2}}{\left[h_{\mathrm{P}}\right]\left(a_{\mathrm{FeO}}\right)^{5 / 2}\left(a_{\mathrm{CaO}}\right)^{2}}\right]
$$

Considerando, na Equação 6, o ferro como solvente e seguindo a lei de Raoult, $\mathrm{aFe} \cong \mathrm{NFe} \cong \mathrm{I}$ e $\mathrm{hp}=\%$ P.fp, tem-se a Equação 7:

$$
-\frac{\Delta G^{0}}{R T}=\ln \left[\frac{\left(a_{\mathrm{Ca}_{2} \mathrm{PO}_{4,5}}\right)}{\left[\% \mathrm{P} . f_{\mathrm{P}}\right]\left(a_{\mathrm{FeO}}\right)^{5 / 2}\left(a_{\mathrm{CaO}}\right)^{2}}\right]
$$

Aplicando as propriedades de logaritmo na Equação 7 obtém-se a Equação 8:

$$
-\frac{\Delta G^{0}}{R T}=\ln \left(a_{C a_{2} P_{4,5}}\right)-\ln [\% P]-\ln \left[f_{P}\right]-\ln \left(a_{F e O}\right)^{5 / 2}-\ln \left(a_{C a O}\right)^{2}
$$

Remanejando os termos da Equação 8, tem-se:

$$
\ln \left(a_{C_{2} P O_{4}}\right)-\ln [\% P]-\ln \left(a_{F_{e O} O}\right)^{5 / 2}=\frac{\Delta G^{0}}{R T}+\ln \left[f_{P}\right]+\ln \left(a_{C a O}\right)^{2}
$$

Aplicando novamente as propriedades do logaritmo na Equação 9, tem-se a Equação 10 :

$$
\ln \left[\frac{\left(a_{\mathrm{Ca}_{2} \mathrm{PO}_{4,5}}\right)}{[\% P]\left(a_{\mathrm{FeO}}\right)^{5 / 2}}\right]=-\frac{\Delta G^{0}}{R T}+\ln \left[f_{P}\right]+2 \ln \left(a_{\mathrm{CaO}}\right)
$$

Sabendo que:

$$
\begin{aligned}
& a_{\mathrm{Ca}_{2} \mathrm{PO}_{4,5}}=\gamma_{\mathrm{Ca}_{2} \mathrm{PO}_{4,5}} \mathrm{~N}_{\mathrm{Ca}_{2} \mathrm{PO}_{4,5}} \\
& a_{\mathrm{CaO}}=\gamma_{\mathrm{CaO}} N_{\mathrm{CaO}} \\
& a_{\mathrm{FeO}}=\gamma_{\mathrm{FeO}} N_{\mathrm{FeO}}
\end{aligned}
$$

Considerando por simplificação que, $\gamma_{\mathrm{Ca}_{2} \mathrm{PO}_{4,5},}, \gamma_{\mathrm{CaO}} e \gamma_{\mathrm{FeO}}$ apresentados nas Equações II, 12 e 13 são constantes, conforme as equações 14 , 15 e 16 :

$$
\begin{aligned}
& N_{\mathrm{Ca}_{2} \mathrm{PO}_{4,5}}=\text { cte. } \% \mathrm{P}_{2} \mathrm{O}_{5} \\
& N_{\mathrm{CaO}}=c t e . \% \mathrm{CaO} \\
& N_{\mathrm{FeO}}=c t e . \% \mathrm{FeO}
\end{aligned}
$$

Logo,

$$
a_{\mathrm{Ca}_{2} \mathrm{PO}_{4,5}}=\text { cte. } \% \mathrm{P}_{2} \mathrm{O}_{5}
$$

$$
a_{\mathrm{CaO}}=c t e . \% \mathrm{CaO}
$$




$$
\begin{aligned}
& \text { Sendo } \% F e_{t}=\operatorname{soma~} F e^{2+} e F e^{3+} \text {, então: } \\
& a_{\mathrm{FeO}}=c t e . \% F e_{t}
\end{aligned}
$$

Substituindo as Equações 17, 18 e 19 em (9), obtém-se a Equação 20:

$$
\ln \left[\frac{\left(\text { cte. } \% P_{2} O_{5}\right)}{[\% P]\left(c t e . F_{t}\right)^{5 / 2}}\right]=-\frac{\Delta G^{0}}{R T}+\ln \left[f_{P}\right]+2 \ln (\text { cte. } \% \mathrm{CaO})
$$

Como $\Delta G^{0}=c t e+c t e . T$, então:

$$
\frac{\Delta G^{0}}{R T}=\frac{c t e}{R T}+\frac{c t e \cdot T}{R T}
$$

Simplificando a equação 21 , tem-se a Equação 22:

$$
\frac{\Delta G^{0}}{R T}=\frac{c t e}{T}+c t e
$$

Substituindo a equação 22 na equação 20, tem-se a Equação 23:

$$
\ln \left[\frac{\left(\text { cte. } \% P_{2} O_{5}\right)}{[\% P]\left(c t e . F e_{t}\right)^{5 / 2}}\right]=-\left(\frac{c t e}{T}+c t e\right)+\ln \left[f_{P}\right]+2 \ln (c t e . \% C a O)
$$

Sabendo que:

- $f_{P}$ é aproximadamente constante;

- a transformação do logaritmo neperiano para logaritmo decimal gera mais uma constante;

- $\ln (\% \mathrm{CaO})$ é função de uma constante: $\ln (\% \mathrm{CaO})=$ cte + cte. $\% \mathrm{CaO}[6]$.

Podem-se agrupar todas as constantes, separando-as em apenas um termo:

$$
\log \left[\frac{\left(\% P_{2} O_{5}\right)}{[\% P]\left(F e_{t}\right)^{5 / 2}}\right]=-\left(\frac{c t e}{T}\right)+c t e . \% C a O+c t e
$$

Finalmente, a partir da Equação 24, obtém-se a seguinte equação para a partição do fósforo, que será definida como a variável resposta conforme a Equação 25.

$$
\log \left[\frac{\left(\% P_{2} O_{5}\right)}{[\% P]\left(\mathrm{Fe}_{t}\right)^{5 / 2}}\right]=\frac{A}{T}+B . \% \mathrm{CaO}+\mathrm{C}
$$

Com o objetivo de melhorar a eficiência do tratamento de desfosforação de ferro-gusa em panela da Siderúrgica Alterosa, otimizando a quantidade de agente desfosforante utilizado durante o tratamento, foi desenvolvido um modelo de previsão de fósforo ao final do tratamento de desfosforação de ferro-gusa líquido em panela, utilizando a ferramenta estatística de regressão linear múltipla.

\section{METODOLOGIA}

No processo de desfosforação da Siderúrgica Alterosa, o ferro-gusa é vazado para uma panela onde é feita a adição do agente desfosforante por meio de uma lança refratária submersa no banho de ferro-gusa. Durante esse processo, é soprado, também, oxigênio, que além de auxiliar na desfosforação, mantém a temperatura do banho, garantindo melhor operacionalidade no tratamento e melhor aspecto visual do ferro-gusa. $O$ produto resultante deste processo é chamado de ferro-gusa nodular especial [7].

As características do ferro-gusa utilizado durante o experimento estão apresentadas na Tabela I:

$O$ agente desfosforante foi produzido na Tecnosulfur $\mathrm{S} / \mathrm{A}$, através de um complexo sistema de moagem com ambiente controlado, que significa que o ambiente interno de moagem está isento de umidade, onde as matérias-primas componentes deste insumo passaram por controle de seleção, pesagem, moagem e homogeneização.

Após o processo produtivo o produto foi enviado à Siderúrgica Alterosa através de carreta silo e descarregado no silo de armazenamento de produto. As especificações, química e física, do agente desfosforante estão apresentadas na Tabela 2.

A Figura 2, mostra de forma esquemática a estação de desfosforação que foi utilizada no estudo.

\section{I Coleta dos Dados para o Desenvolvimento e Validação do Modelo}

Para realização do presente estudo foram coletados dados de 150 corridas do processo de desfosforação em panela. $O$ ferro-gusa utilizado no experimento foi produzido a partir de um alto-forno que utiliza carvão vegetal como combustível. O ferro-gusa produzido foi vazado em uma panela com capacidade de 35 toneladas e após encaminhado para a estação de pré-tratamento. Após o tratamento a escória gerada através do processo de remoção do Fósforo foi removida e o ferro-gusa tratado foi levado para o lingotamento.

Tabela I. Características do ferro-gusa utilizado durante o experimento

\begin{tabular}{ccc}
\hline Características do ferro-gusa & Mínimo (\%) & Máximo (\%) \\
\hline Silício $(\mathrm{Si})$ & 0,07 & 0,48 \\
Fósforo $(\mathrm{P})$ & 0,090 & 0,150 \\
Temperatura $\left({ }^{\circ} \mathrm{C}\right)$ & 1.243 & 1.329 \\
Peso de ferro-gusa $(\mathrm{t})$ & 22,82 & 34,95 \\
\hline
\end{tabular}

Tabela 2. Especificação química e física do Agente Desfosforante utilizado no experimento

\begin{tabular}{ccc}
\hline Compostos & Mínimo (\%) & Máximo (\%) \\
\hline $\mathrm{CaO}$ & 35,00 & 52,00 \\
$\mathrm{CaF} 2$ & 0,00 & 12,00 \\
$\mathrm{Fe} 2 \mathrm{O} 3$ & 35,00 & 52,00 \\
\cline { 2 - 3 } & Mínimo (mm) & Máximo (mm) \\
\cline { 2 - 3 } Granulometria & 0,00 & 2,00 \\
\hline
\end{tabular}




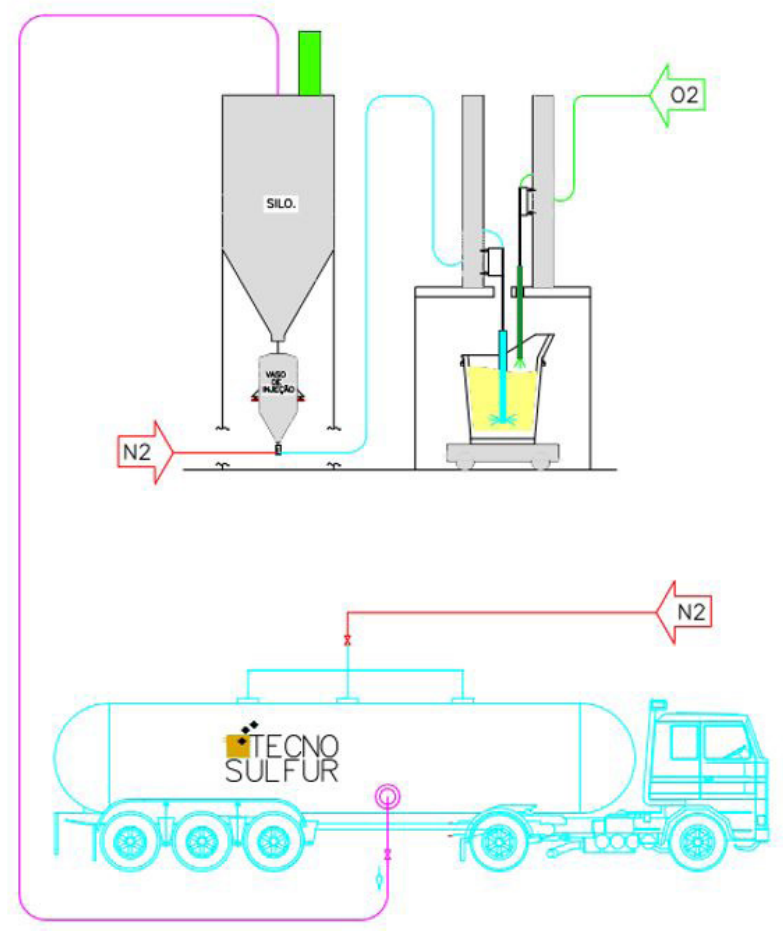

Figura 2. Figura esquemática da estação de desfosforação de gusa em panela. do modelo:

Dados coletados para o desenvolvimento e validação

- Lote I: Inicialmente foram acompanhadas e coletados dados de 105 corridas para a construção do modelo de previsão da partição de fósforo entre o metal e a escória;

- Lote 2: Após o desenvolvimento do modelo foram acompanhadas e coletados dados de 45 corridas para a validação do modelo de previsão da partição de fósforo entre metal e a escória;

- Lote 3: Após validado o modelo de predição da partição de fósforo entre o metal e a escória, foram utilizados 150 dados das corridas utilizadas nas etapas anteriores para a validação do modelo de predição de Fósforo (\%P) ao final do tratamento do ferro-gusa.

\section{RESULTADOS}

O desenvolvimento do modelo de previsão de Fósforo seguiu o conceito de construção de um modelo de regressão linear múltipla desenvolvido, que é composto por quatro fases [8]:

I. Coleta e preparo dos dados;
2. Redução do número de variáveis explicativas Foi utilizado a metodologia de regressão de melhores subconjuntos que é um procedimento automático que identifica os modelos de regressão com melhor ajuste com os preditores especificados;

3. Refinamento e seleção do modelo - Foi avaliado a normalidade dos resíduos, homoscedasticidade e a independência dos resíduos, apresentados na Figura 3;

4. Validação do modelo - O melhor método de validação de um modelo de regressão consiste na coleta de novos dados com o objetivo de avaliar se o modelo desenvolvido é também aplicável aos novos dados e não apenas ao conjunto de dados utilizados para o ajuste [8].

Durante o acompanhamento das corridas experimentais foram coletados os seguintes dados: Peso do ferro-gusa ( $t$ ); Teor inicial de silício no ferro-gusa, (\%); Teor inicial de fósforo no ferro-gusa, (\%); Teor final de fósforo no ferro-gusa, (\%); Temperatura inicial do ferro-gusa, $\left({ }^{\circ} \mathrm{C}\right)$; Consumo de oxigênio, $\left(\mathrm{Nm}^{3}\right)$; Consumo específico de agente desfosforante, $(\mathrm{kg} / \mathrm{t})$; Taxa de injeção de agente desfosforante, $(\mathrm{kg} / \mathrm{min})$. Utilizando os dados coletados relacionados no lote I e com - auxílio do Software Comercial Minitab I 7 foi desenvolvido uma equação de regressão linear múltipla capaz de prever a partição de fósforo, conforme a Equação 26.

$$
\begin{aligned}
& \log \left[\frac{\left(\% P_{2} O_{5}\right)}{[\% P]\left(F e_{t}\right)^{5 / 2}}\right]=-2,59+0,01112 x F G- \\
& 244 x P i-205,6 x S i+\frac{11110}{T}+0,01596 x C E
\end{aligned}
$$

Onde:

$F G=$ Peso do ferro - gusa $(\mathrm{t})$;

$P i=$ Teor inicial de Fósforo do ferro - gusa $(\%)$;

$S i=$ Teor de Silício inicial do ferro - gusa $(\%)$;

$T=$ Temperatura inicial do ferro - gusa $(\mathrm{K})$;

C.E. $=$ Consumo específico do agente desfosforante $\left(\frac{\mathrm{kg}}{\mathrm{t}}\right)$.

Os dados industriais das corridas acompanhadas relacionadas como lote 2 foram utilizados para validação do modelo de predição da partição de fósforo conforme os resultados apresentados abaixo:

Utilizando o modelo de regressão apresentado na Equação 26 foi plotado o gráfico de validação do modelo apresentado na Figura 4 e pode-se concluir que o modelo de regressão desenvolvido para a previsão da partição do fósforo entre o metal e a escória está apropriado para a utilização, visto que, o valor de (R2 e R2(aj)) e S encontrados no teste de validação com os novos dados do lote 2 estão próximos aos valores encontrados no lote I no desenvolvimento do modelo, que foram $66,4,65,6$ e 0,109 , respectivamente. 
Gráfico de Probabilidade Normal

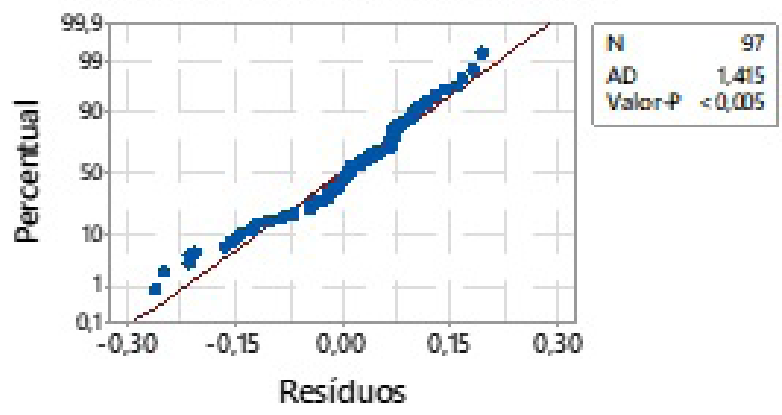

Histograma

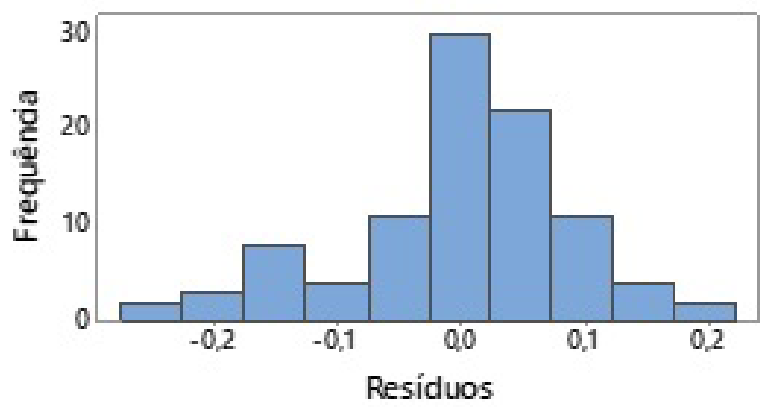

Versus Ajustados

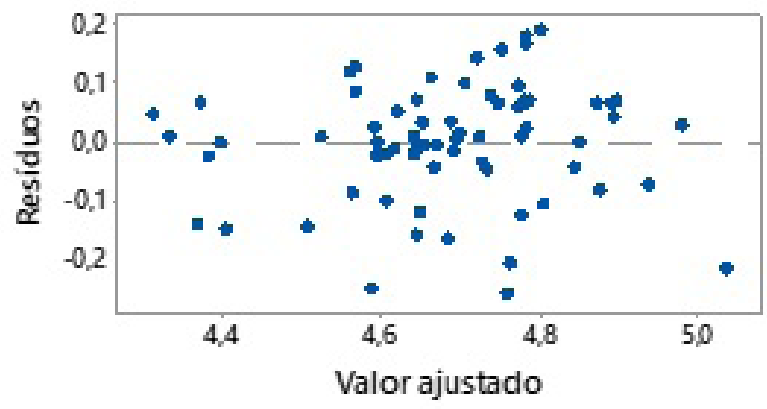

Versus Ordem

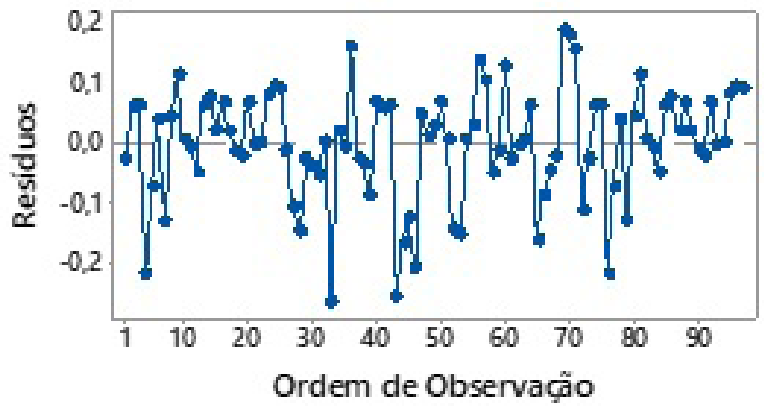

Figura 3. Resultado da análise dos resíduos do desenvolvimento da equação de regressão.

\section{DISCUSSÃO}

Através do balanço de massa do fósforo no tratamento de desfosforação e com o auxílio da partição de fósforo, variável resposta, da Equação 26 foi possível desenvolver o modelo de predição de fósforo ao final do tratamento, conforme a Equação 27.

- Ferro-gusa: Massa $_{\text {Ferro-gusa }} \times \frac{\% P_{\text {final }}}{100}$

- Escória: Massa $_{\text {escória }} \times\left(\frac{\% P_{2} O_{5}}{100} \times \frac{\text { Peso Molecular }_{P}}{{\text { Peso } \text { Molecular }_{P_{3} O_{5}}}_{1}}\right)$

$$
M_{F G} x \frac{\% P_{\text {inicial }}}{100}=M_{F G} x \frac{\% P_{\text {final }}}{100}+M_{E S C} x\left(\frac{\% P_{2} O_{5}}{100} x \frac{2 x P M_{P}}{P M_{P_{3} O_{5}}}\right)
$$

Substituindo a Equação 26 em (27) para obter a Equação 28 para a previsão do fósforo ao final do tratamento do ferro-gusa.

$$
\% P=\frac{M_{F G} x \frac{\% P_{\text {inicial }}}{100}}{\frac{M_{F G}}{100}+\frac{M_{E S C} x\left(\% F e_{t}\right)^{5 / 2} \times 10^{-2,59+0,01112 x F G-244 x P i-205,6 x S i+\frac{1110}{T}+0,01596 x C E}}{100}\left(\frac{2 x P M_{P}}{P M_{P_{3} O_{5}}}\right)}
$$


Para avaliação e validação do modelo de previsão do teor de fósforo ao final do tratamento de desfosforação em panela, foram utilizados os dados o lote 3.

Foram plotados os gráficos boxplot dos dados do lote 3 conforme foi realizado nos dados dos lotes I e 2, para verificar a ocorrência de outliers nos dados que pudessem interferir na avaliação no modelo de previsão do teor de fósforo final no tratamento de desfosforação em panela. De acordo com as Figuras 4 e 5 foram encontrados 2 outliers, porém, foram verificados e não estão interferindo no resultado do modelo e por isso não foram desconsiderados das análises.

O estudo de regressão linear é extraído de varáveis contínuas, portanto devem tender para uma distribuição contínua. Assim as variáveis resposta e explicativas devem ser testadas quanto a sua normalidade. Para o estudo foi aplicado, com o auxílio do Minitab 17, o teste de normalidade de Anderson-Darling. Para determinar se as variáveis estão dentro da normalidade esperada deve-se avaliar o Valor-p da variável e como o Valor-p das amostras do lote 3 para o teor de fósforo final observado e o teor de fósforo final predito são menores que o nível de significância de 0,05 o teste afirma que os dados seguem uma distribuição normal. A Tabela 3 apresenta a descrição das variáveis explicativas utilizadas no modelo.

O resultado da Figura 5 mostra que o percentual de explicação do modelo de predição de teor de fósforo ao final do tratamento de desfosforação de ferro-gusa em panela foi acima de $83,0 \%$ sendo considerado um excelente resultado.

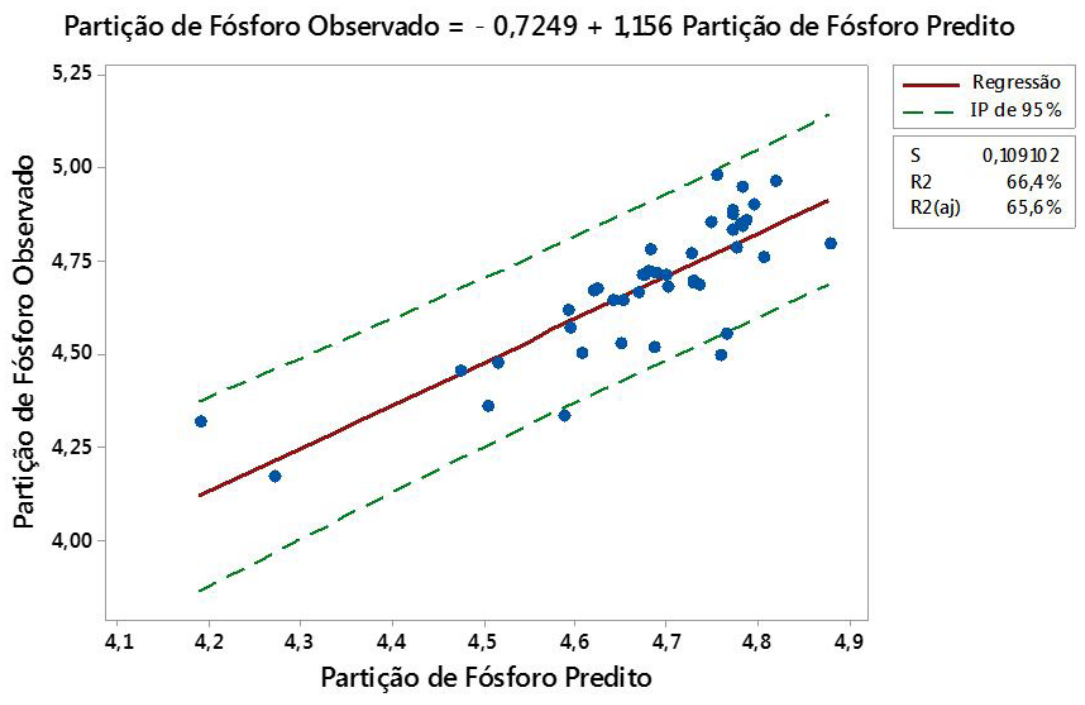

Figura 4. Resultado do ajuste do modelo preditivo da partição de fósforo através da Equação 26.

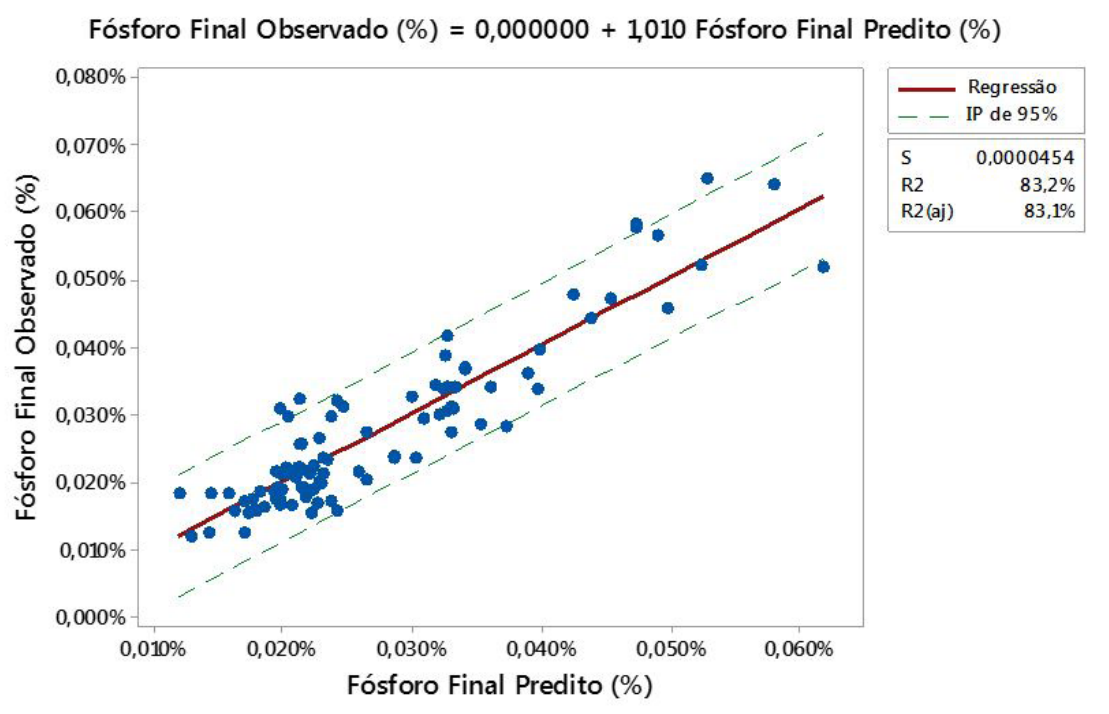

Figura 5. Resultado do ajuste do modelo preditivo do percentual de fósforo $P$ utilizando Equação 28. 
Tabela 3. Descrição das variáveis que fazem parte do cálculo do percentual de fósforo

\begin{tabular}{|c|c|c|}
\hline Variáveis & Mediana (QI;Q3) & Média \pm DP \\
\hline Ferro-Gusa & $32,8 \mathrm{I}(3 \mathrm{I}, 85 ; 35,05)$ & $32,96 \pm 2,84$ \\
\hline Silício inicial & $0,003(0,002 ; 0,003)$ & $0,003 \pm 0,001$ \\
\hline Fosforo inicial & $0,0010(0,00096 ; 0,0012)$ & $0,001 \pm 0,0001$ \\
\hline Fosforo final & $0,0002 \mid(0,000|8 ; 0,0003|)$ & $0,0003 \pm 0,0001$ \\
\hline Temperatura inicial & $1288,5(|274,0 ;| 305,0)$ & $1289,7 \pm 19,2$ \\
\hline Consumo específico & $36,90(31,67 ; 44,02)$ & $36,67 \pm 7,69$ \\
\hline
\end{tabular}

\section{CONCLUSÃO}

O modelo desenvolvido através do conceito de regressão linear múltipla prevê de forma precisa o teor de fósforo final no ferro-gusa após o tratamento de desfosforação em uma estação em panela. Esse conceito será utilizado para calcular a quantidade de agente desfosforante que deve ser utilizado em um tratamento de desfosforação, para um determinado teor de fósforo final objetivado no ferro-gusa, considerando o peso, teor de fósforo, silício e temperatura inicial do ferrogusa a ser tratado.

\section{Agradecimentos}

Os autores agradecem à Tecnosulfur Sistema de Tratamento de Metais Líquidos S/A e a Siderúrgica Alterosa S/A e também ao PROEX CAPES e FAPEMIG pelo suporte financeiro ao PPGEM.

\section{REFERÊNCIAS}

I Instituto Aço Brasil [página da internet]. [acesso em 2 jun. 2016]. Disponível em: http://www.acobrasil.org.br

2 Bannenberg N, Lachmund $\mathrm{H}$. Metallurgical procedures for achieve very low phosphorus contents. La Revue de Metallurge. 1994;9I(7-8): 1043-1054.

3 Kitamura, S. Treatise on process metallurgy. Toronto: Elsevier; 20I4. Vol. 3.

4 Almdeida L. Desenvolvimento de um modelo de previsão de fósforo para convertedor LD da V\&M do Brasil. [dissertação]. Universidade Federal de Minas Gerais, Belo Horizonte; 2005.

5 Healy GW. A new look at phosphorus distribution. Journal of The Iron and Steel Institute. 1970;208(7):664-668.

6 Kitamura S. Treatise on process metallurgy. Vol. 3. Amesterdã: Elsevier; 2014.

7 Siderúrgica Alterosa [página da internet]. [acesso em I5 maio 2016]. Disponível em: http://www.grupoalterosa.ind. br/alterosa.php?conteudo=informativo

8 Werkema MCC, Aguiar S. Análise de regressão: como entender o relacionamento entre variáveis de um processo. Belo Horizonte: Fundação Christiano Ottoni, Universidade Federal de Minas Gerais. 1996. 31 I p. (Série Ferramentas da Qualidade, vol. 7).

Recebido em: 28 Jan. 2017

Aceito em: 13 Mar. 2017 